\title{
Digital Image Restoration in Matlab: A Case Study on Inverse and Wiener Filtering
}

\author{
Mohammad Mahmudur Rahman Khan $^{1 *}$, Shadman Sakib ${ }^{2 \#}$, Rezoana Bente Arif ${ }^{2 @}$, and Md. Abu Bakr Siddique ${ }^{2 \$}$ \\ ${ }^{I}$ Dept. of ECE, Mississippi State University, Mississippi State, MS 39762, USA \\ ${ }^{2}$ Dept. of EEE, International University of Business Agriculture and Technology, Bangladesh \\ mrk303@msstate.edu ${ }^{*}$, sakibshadman15@gmail.com”, rezoana@iubat.edu ${ }^{\circledR}$, absiddique@iubat.edu \\ Corresponding Author: absiddique@iubat.edu
}

\begin{abstract}
In this paper, at first, a color image of a car is taken. Then the image is transformed into a grayscale image. After that, the motion blurring effect is applied to that image according to the image degradation model described in equation 3. The blurring effect can be controlled by $a$ and $b$ components of the model. Then random noise is added in the image via Matlab programming. Many methods can restore the noisy and motion blurred image; particularly in this paper Inverse filtering as well as Wiener filtering are implemented for the restoration purpose. Consequently, both motion blurred and noisy motion blurred images are restored via Inverse filtering as well as Wiener filtering techniques and the comparison is made among them.
\end{abstract}

Keywords-Color image, grayscale image, motion blurring, random noise, inverse filtering, Wiener filtering, restoration of an image.

\section{INTRODUCTION}

In digital image processing, image restoration is an essential approach used for the retrieval of uncorrupted, original image from the blurred and noisy image $[1,2]$ because of motion blur, noise, etc. caused by environmental effects [3] and camera misfocus. Image blur may occur for many reasons such as motion blur which is due to the sluggish camera shutter speed comparative to the instantaneous motion of the targeted object [4]. The image also may subject to several forms of noises such as Poisson noise, Gaussian noise, etc. Poisson noise is controlled by signal and it is associated with the low light sources owing to photon counting statistic [4]. In contrast, the reason of Gaussian noise is because of electronic components and broadcast transmission effects [4]. In short, the term image restoration is an inverse process [5] by which the uncorrupted, original image can be recovered from the degraded form of the actual image [6]. There are many useful applications of digital image restoration in several fields including the area of astronomical imaging, medical imaging, media and filmography, security and surveillance videotapes, law enforcement and forensic science, image and video coding, centralized aviation assessment procedures [7], uniformly blurred television pictures restoration [8], etc. Several algorithmic techniques such as Artificial Neural Network [9], Convolutional neural Network [10], and K-nearest Neighbors [11] can also be applied in image processing techniques such as segmentation, thresholding and filtering. The technique used in image restoration is known as filtering which suppresses or removes unwanted components or features from the images. The most popular filtering techniques are used in image restoration in recent times are inverse filtering and Wiener filtering [12].
Inverse filter is a handy technique for image restoration if a proper degradation function can be modeled for the corrupted image. The performance of the inverse filter is quite right when the noise does not corrupt images, but in the presence of noise in the images, performance degrades significantly as high pass inverse filtration cannot eliminate noise properly because noise tends to be high frequency.

Wiener filter is incorporated with low pass filter together with high pass filter; as a result, it works actively in the existence of additive noise within the image. It performs deconvolution operation (high pass filtering) to invert motion blurring and also perform compression operation i.e. (low pass filtering) to eliminate the additive noise. Furthermore, in the process of inverting motion blurring and noise elimination, Wiener filter diminishes the overall mean square inaccuracy between the original and the output image of the filtration.

In this paper, the implementation of inverse filtering and Wiener filtering are analyzed for image restoration. Inverse filtering is applied into a motion blurred car image at first, and then wiener filtering is also used to the same image. After that, inverse and Wiener filtering are performed on the same motion blurred car image with additive noise. Finally, the comparison is made between inverse and Wiener filtering regarding their performances in restoring motion blurred images with and without additive noise.

\section{LITERATURE REVIEW}

Over the past two decades, the technique of image processing has taken its place into every aspect of today's technological society. In digital image processing, there are a variety of essential steps involved such as image enhancement, pre-processing of images, image segmentation, image restoration and reconstruction of images etc. Among them, image restoration plays a vital role in today's world. It has several fields of applications in the areas of astronomy, remote sensing, microscopy, medical imaging, satellite imaging, molecular spectroscopy, law enforcement, and digital media restoration etc. Image restoration is very challenging as there is a lot of interference and noise in the environment like Gaussian noise, multiplicative noise, and impulse noise etc, inclusive of the camera such as wide angle lens, long exposure times, wind speed and degradation, blurring such as uniform blur, atmospheric blur, motion blur, and Gaussian blur etc. However, there are various methods of image restoration in the domain of image processing, for instance, Median filter, Wiener filtering, inverse filtering, Harmonic mean filter, Arithmetic mean filter, Max filter, and Maximum Likelihood (ML) method etc. Among these restoration methods, Wiener and inverse filtering method is the 
simplest and advantageous method for overcoming the current restoration challenges mentioned above.

Stephen et al. outlined the restoration and reconstruction process from overlapping images of the multiple and same scene which is subjected to user-defined and data availability constraints on the support for the spatial domain process [13]. Michael et al. proposed an approach for outof-focus blur and projector blur to reduce the image blur [14]. Yu et al. introduced an algorithm for the restoration of distorted and noisy images degraded by impulse and Gaussian noises [15]. Restoration of digitized photographs can be made by using multi-resolution texture synthesis and image imprinting [16]. Image restoration based on neural networks mainly focuses on spatial variation in terms of changeable regularization parameter for adaptively training the weights [17]. Moreover, the image can be restored by using a novel adaptive $\mathrm{k}$-th nearest neighbor $(\mathrm{KNN})$ strategy variant of the mean shift by knowing its neighbor [18], unsupervised, information-theoretic, adaptive filtering (UINTA) which improves the pixel intensities [19]. In another approach, the image can be restored from the mixed noise through minimization approach [20]. Recently, image restoration based on Convolutional Neural Network (CNN) achieved an encouraging implementation such as deep networks which performs non-local color image denoising [21], model-based optimization method to solve the various inverse problems like deblurring [22]. Moreover, the image can be restored by using the iterative method using denoising algorithm which provides a solution for the linear inverse problem [23].

The median filter is complex to execute as well as it's very time-consuming. Max filter cannot find the black or dark colored pixel of an image. When there is a need of sharp edges in the output, the arithmetic mean filter cannot provide the sharp edges rather it blurs the edges. ML method is sensitive to noise as the reversal of the imaging equation. Moreover, for pepper noise harmonic mean filter does not work well. After all those drawbacks of image restoration process mentioned above, Wiener and inverse filtering method are prominent and beneficial. The mean square error between the uncorrupted image is minimized by using the Wiener filter also it is not sensitive to noise. Inverse filtering is the prominent and simplest method to restore the image in the existence of noise and blur. In parallel, both wiener, and inverse filtering are used to retrieve the noisy and motion blurred images.

\section{FUNDAMENTALS OF IMAGE RESTORATION}

Image restoration is a restoring or recovering process of a degraded image by utilizing some prior knowledge of degradation method which has degraded the image. So the image restoration process involves the estimation of the deteriorated model as well as the relevance of the inverse filtering to restore or retrieve the original image [24]. Although the reconstructed image may not be the exact form of the original image, it will be the approximation of the original image. Figure 1 below shows a fundamental model of image degradation and restoration procedure.

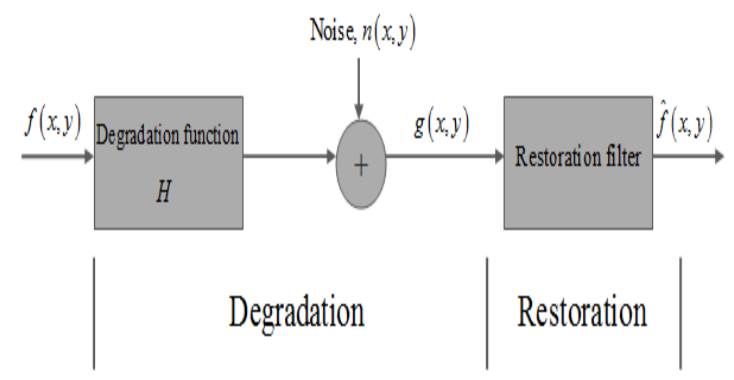

Fig. 1. The fundamental outline of image degradation and restoration procedure

In spatial domain, the degradation of the original image can be modeled as [25]:

$$
g(x, y)=h(x, y) * f(x, y)+n(x, y)
$$

Where,

$(x, y)=$ detached pixel coordinates of the image frame.

$f(x, y)=$ Original image

$g(x, y)=$ Degraded image

$h(x, y)=$ Image degradation function

$n(x, y)=$ Ad-on noise

As convolution operation within the spatial domain corresponds to multiplication in the frequency domain, the equation 1 can be rewritten as:

$$
G(u, v)=H(u, v) \times F(u, v)+N(u, v)
$$

Now, Motion blur is present when there exists comparative motion in the midst of the recording device and the scene (object). However, the types of blur may be in the appearance of a translation, rotation, and scaling, or some combinations of these. Here only the critical case of a global translation will be considered.

Let's pretend the scene to be recorded interprets comparative to the camera at constant velocities $a$ and $b$ along the directions of $\mathrm{x}$ and $\mathrm{y}$ during the exposure time $\mathrm{T}$. The frequency domain degradation function can be simplified as [26]:

$H(u, v)=\frac{\sin (\pi(u a+v b) T)}{\pi(u a+v b)} e^{-j \pi(u a+v b) T}$

Image restoration process can be subdivided into two classes:

- Deterministic methods are applicable to images with a small amount of noise and a familiar degradation function.

- Stochastic techniques are to restore images according to some stochastic criterion. 


\section{A. The Basics of Inverse Filtering}

Like any other unsupervised methods such as Fuzzy CMeans [27] and ADBSCAN [28] clustering, inverse filtering is also unsupervised. The basic image restoration model for inverse filtering is exposed in figure 2 .

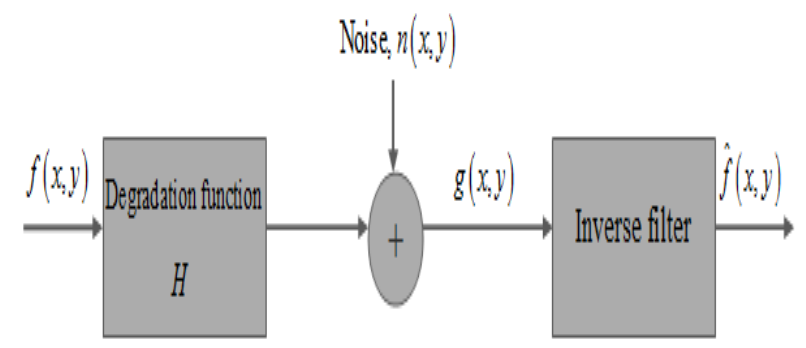

Fig. 2. Image restoration model (Inverse filtering)

When the degradation function $\mathrm{H}(\mathrm{u}, \mathrm{v})$ is identified, the image can be returned to normal state by:

$$
\hat{F}(u, v)=\frac{G(u, v)}{H(u, v)} \ldots
$$

Now, in our case, we have added noise after implementing the motion blurring effect. Hence we have used the following formula to restore the original image:

$$
\hat{F}(u, v)=F(u, v)+\frac{N(u, v)}{H(u, v)}
$$

Since, the function of $\mathrm{N}(\mathrm{u}, \mathrm{v})$ is random whose Fourier transform is generally unfamilier, it is impossible to retrieve $\mathrm{F}(\mathrm{u}, \mathrm{v})$ accurately. The impact of noise is noteworthy for frequencies where $\mathrm{H}(\mathrm{u}, \mathrm{v})$ has a tiny magnitude. In reality, $\mathrm{H}(\mathrm{u}, \mathrm{v})$ usually decreases in size much more rapidly than $\mathrm{N}(\mathrm{u}, \mathrm{v})$ and thus the noise effect $\mathrm{N}(\mathrm{u}, \mathrm{v}) / \mathrm{H}(\mathrm{u}, \mathrm{v})$ could take over the entire restoration result.

\section{B. The Basics of Wiener Filtering}

The basic image restoration model for Wiener filtering is modeled in figure 3 .

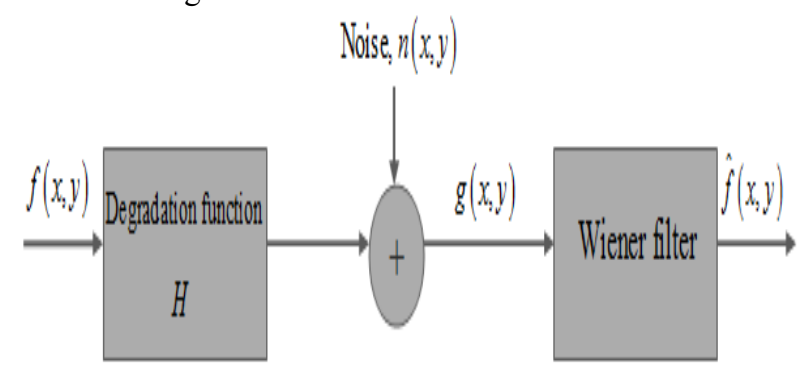

Fig. 3. Image restoration model (Wiener filtering)

Wiener filter exploits the previous knowledge of the spectral properties of the original signal and the noise and linear time-invariant rule to produce an output as close to the original image as feasible. In wiener filtering, it is presumed that the signal and noise are static linear stochastic processes with familiar spectral properties [29, 30]. Wiener filter tries to reconstruct the degraded image by minimizing an error function as designed by the following equation:

$$
M S E=E\left[\{f(x, y)-\hat{f}(x, y)\}^{2}\right]
$$

Where,

$M S E$ = Mean square error

$E[]=$. The expectation operation

$\hat{f}(x, y)=$ Restored image

The Wiener filter is to locate an approximation $\widehat{f}(x, y)$ of the original image $f(x, y)$ so as to the mean square error between them is minimized. Wiener filter is represented as $L(u, v)$ as shown below [31, 32]:

$$
\begin{aligned}
L(u, v) & =\frac{H^{*}(u, v) S_{f}(u, v)}{|H(u, v)|^{2} S_{f}(u, v)+S_{n}(u, v)} \\
& =\frac{H^{*}(u, v)}{|H(u, v)|^{2}+\frac{S_{n}(u, v)}{S_{f}(u, v)}} \ldots \ldots(7)
\end{aligned}
$$

Again,

$$
\begin{aligned}
L(u, v) & =\frac{H^{*}(u, v) S_{f}(u, v)}{|H(u, v)|^{2} S_{f}(u, v)+S_{n}(u, v)} \\
& =\frac{1}{H(u, v)} \frac{|H(u, v)|^{2}}{|H(u, v)|^{2}+K} \ldots . .(8)
\end{aligned}
$$

Where,

$K=\frac{S_{n}(u, v)}{S_{f}(u, v)}$

$S_{f}(u, v)=$ Power spectrum of the original image

$S_{n}(u, v)=$ Noise power spectrum

Here, $\mathrm{K}$ is the inverse of SNR. The image and noise are considered as arbitrary processes. The Wiener filter can generate optimal estimate only if such stochastic processes are stationary Gaussian. These situations are not typically satisfied for real images. So the restored image can be expressed as:

$$
\hat{f}(u, v)=L(u, v) G(u, v)
$$




\section{RESULTS AND DISCUSSION}

For this paper, the following color image of a car is used as made known in figure 4 .

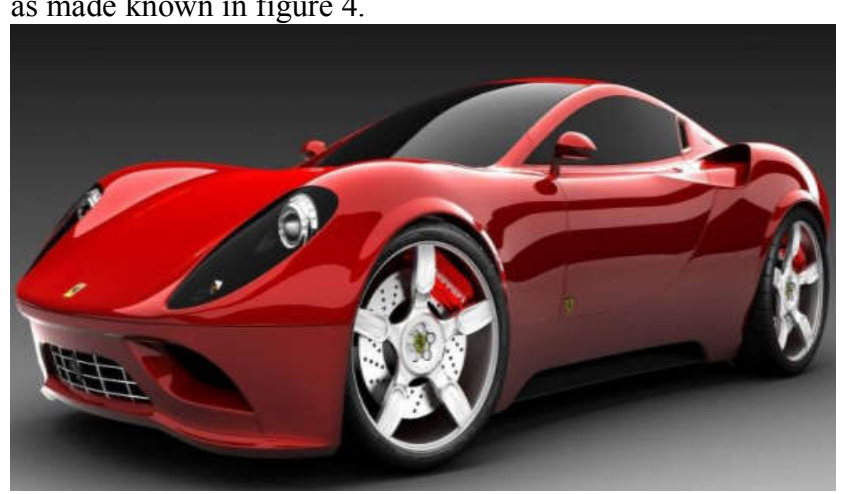

Fig. 4. The color image of a car

Then the image is transformed into a grayscale image in Matlab. The grayscale image is made known in figure 5 below.

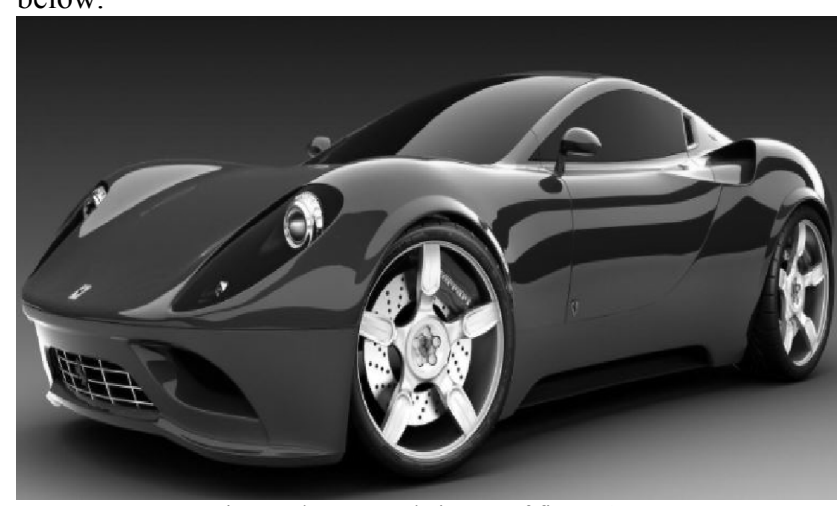

Fig. 5. The grayscale image of figure 1

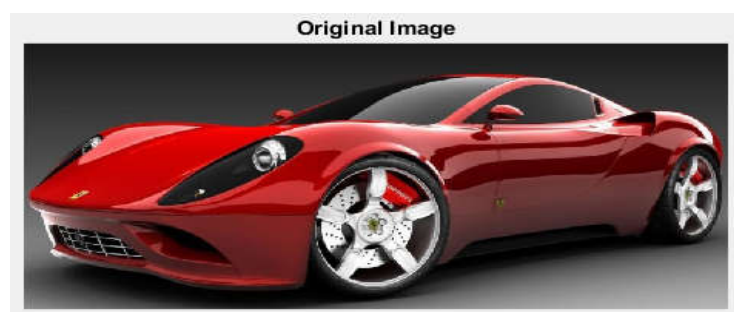

Motion Blurred Image

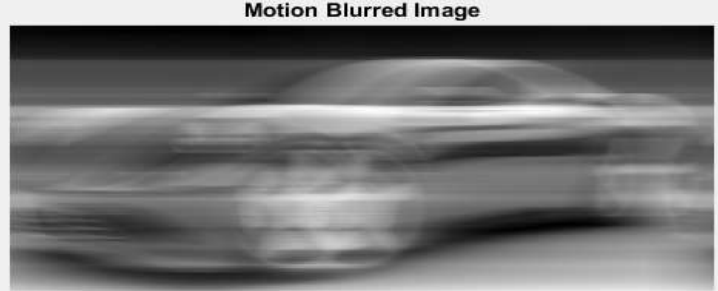

Now, the first task was to insert motion blur effect into the image according to equation (1). After doing so, the resulted image is represented in figure 6 .

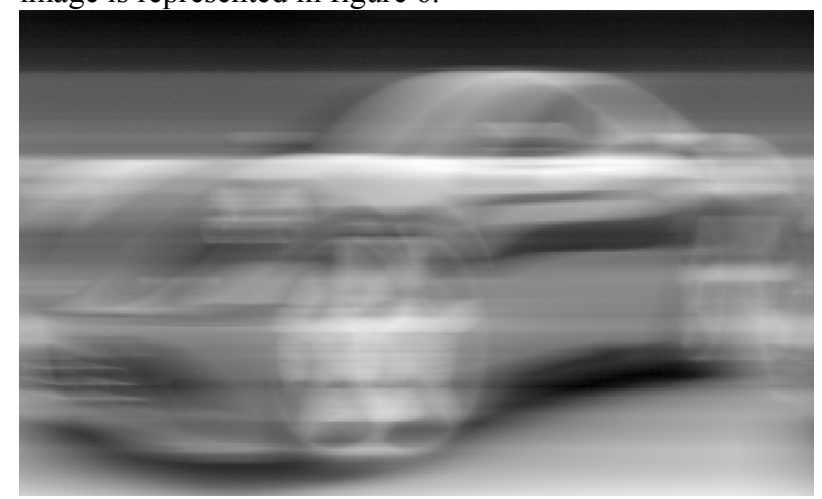

Fig. 6. Grayscale car image with motion blur effect

The effect of the motion blur can be controlled by a and $b$ components of the model.

After applying the blur to the image inverse and, Wiener filterings are implemented to restore the image.

\section{A. The Results of Inverse Filtering}

For inverse filtering, if we do not add any noise after the motion blur, then we can restore the same image before motion blur. The figure 7 below shows the effectiveness of inverse filtering without any noise.

Now, if we add some random noise to the image, then the filter performance degrades to some extent. The consequence of noise on the performance of inverse filtering is made known in figure 8 . In figure 8 , though the inverse filter is capable of inverting the effect of motion blur, it is not able to nullify the effect of noise. Here, $a=0.0001$ and $\mathrm{b}=0.1$.
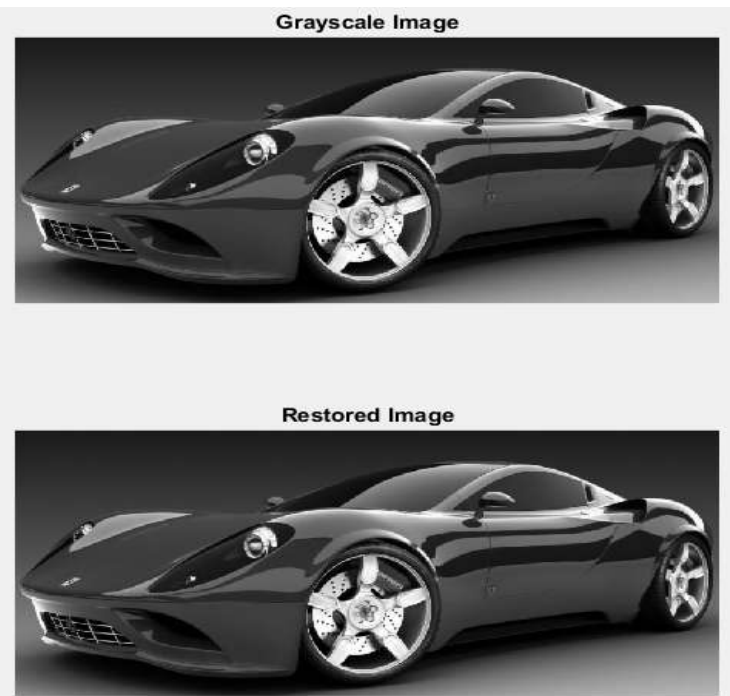

Fig. 7. Restoration of motion blurred car image by inverse filtering 

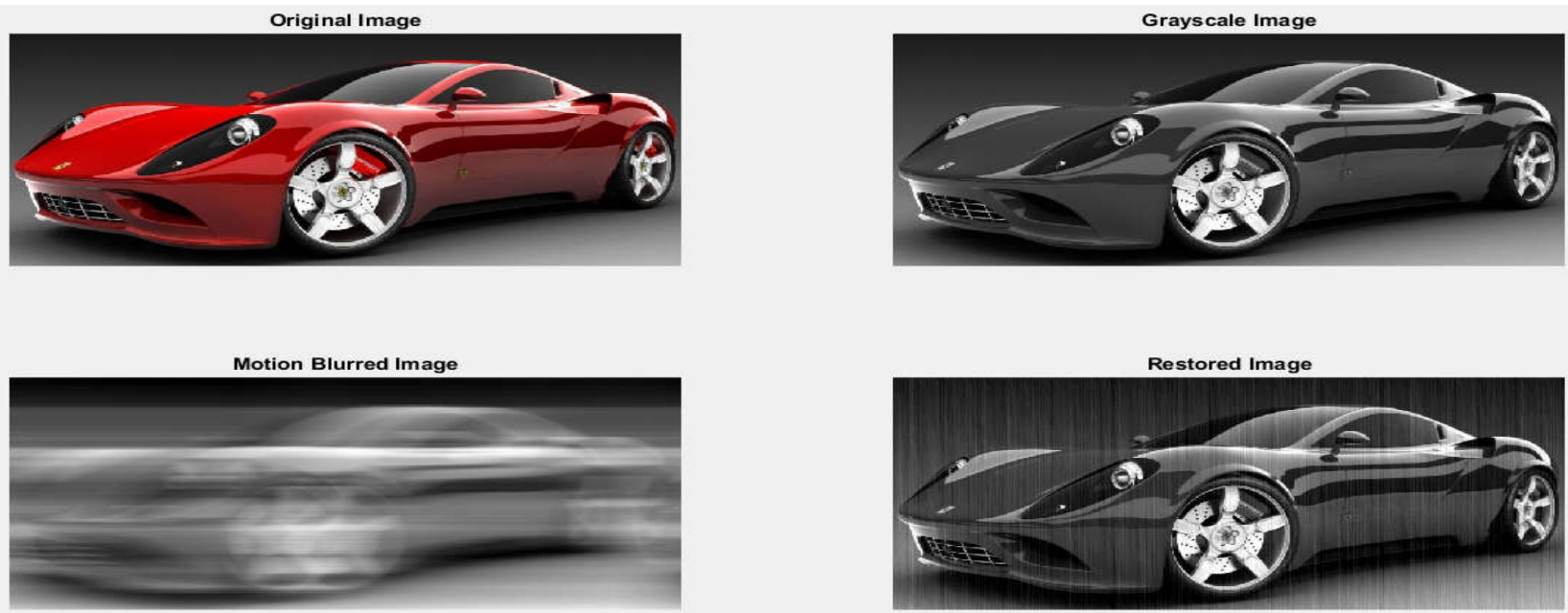

Fig. 8. Restoration of noisy motion blurred car image by inverse filtering

\section{B. The Results of Wiener Filtering}

The Wiener filter has a ' $\mathrm{K}$ ' component which is inverse to the SNR. Now, if the noise power is zero, which means no noise, then the Wiener can restore the exact image which was corrupted by motion blur effect. In the following case, we have considered zero noise power, and figure 9 shows the performance of the Wiener filter.

Original Image

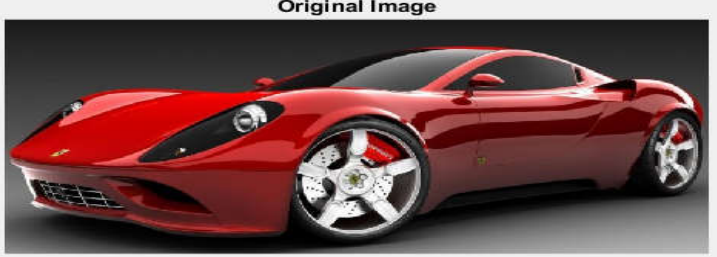

Motion Blurred Image

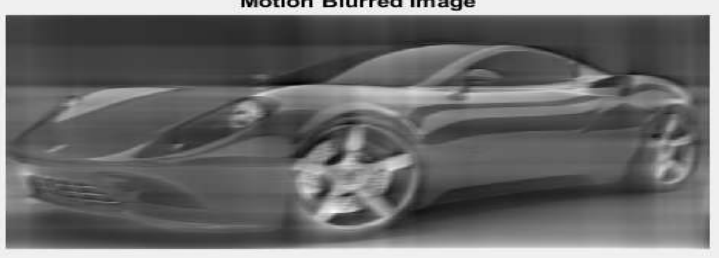

Fig. 9. Restoration of motion blurred car image by Wiener filtering
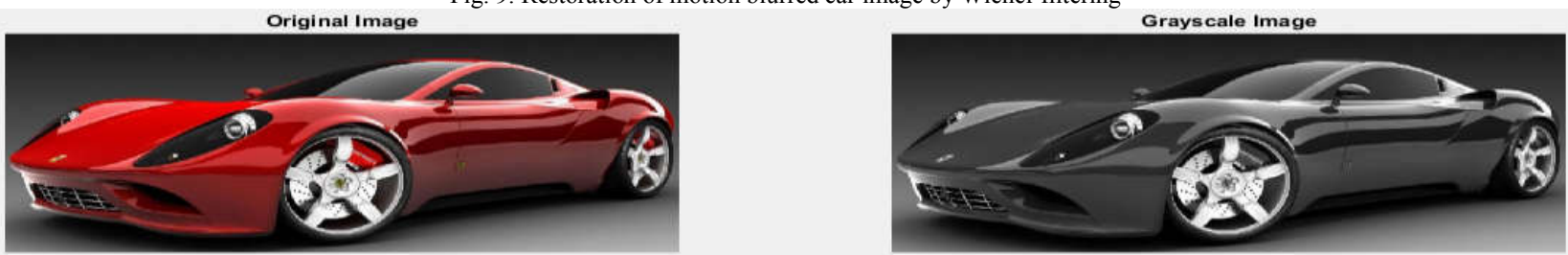

Motion Blurred Image
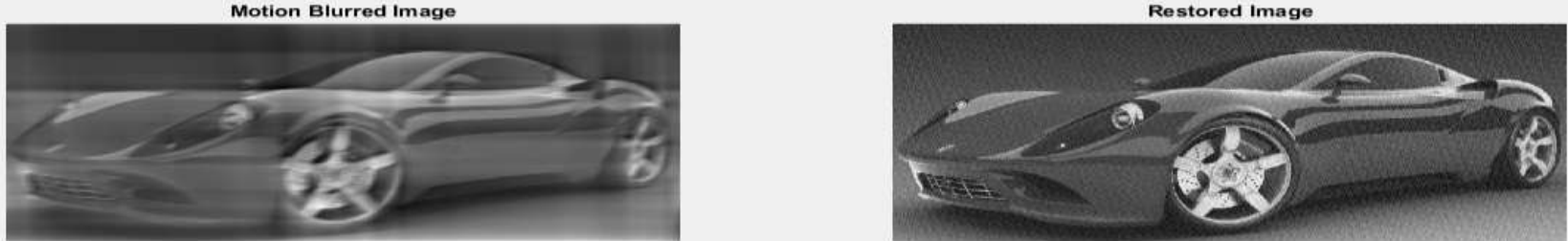

Here, the restored image is almost exactly similar to the image before motion blur. However, if we change the $\mathrm{k}$ to 0.01 , then there would be $1 \%$ noise added after the motion blur effect. If then we apply wiener filter, we will get the following result as represented in figure 10. It is observed that the Wiener filter is reversing the effect of motion blur, but still, there is some noise remaining in the picture.

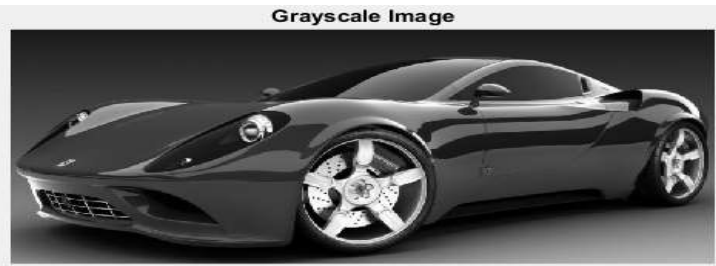

Restored Image

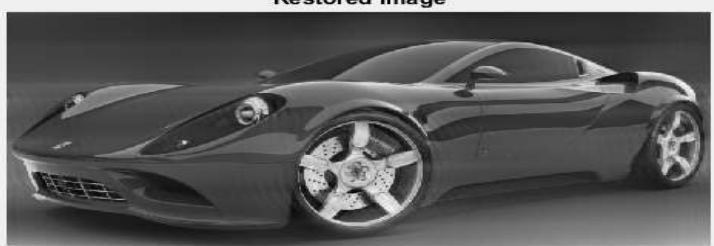

Fig. 10. Restoration of noisy motion blurred car image by Wiener filtering 


\section{CONCLUSION}

This paper presents inverse and wiener filterings' practical implementation on some images for image restoration. It is observed that both inverse and Wiener filtering work quite well in the absence of noise in restoring original image from its degraded version. But in the existence of additive noise wiener filtering works better for restoration purpose compared to inverse filtering. In subsequent works of this series, some other improved filtering techniques for image restoration will be discussed.

\section{REFERENCES}

[1] M. Trimeche, et al., "Multichannel image deblurring of raw color components," in Computational Imaging III, 2005, pp. 169-179.

[2] L. Yang, "Image Restoration from a Single Blurred Photograph," in Information Science and Control Engineering (ICISCE), 2016 3rd International Conference on, 2016, pp. 405409.

[3] T. F. Chan and J. J. Shen, Image processing and analysis: variational, PDE, wavelet, and stochastic methods vol. 94: Siam, 2005

[4] M. R. Banham and A. K. Katsaggelos, "Digital image restoration," IEEE signal processing magazine, vol. 14, pp. 2441, 1997.

[5] S. H. Lee, et al., "Directional regularisation for constrained iterative image restoration," Electronics letters, vol. 39, p. 1642 2003.

[6] A. Murli, et al., "The wiener filter and regularization methods for image restoration problems," in Image Analysis and Processing, 1999. Proceedings. International Conference on, 1999, pp. 394-399.

[7] T. J. Kostas, et al., "Super-exponential method for blur identification and image restoration," in Visual Communications and Image Processing'94, 1994, pp. 921-930.

[8] Z. Liu and J. Xiao, "Restoration of blurred TV picture caused by uniform linear motion," Computer vision, graphics, and image processing, vol. 43, p. 279,1988

[9] Md. Abu Bakr Siddique, et al., "Study and Observation of the Variations of Accuracies for Handwritten Digits Recognition with Various Hidden Layers and Epochs using Neural Network Algorithm," in 2018 4th International Conference on Electrical Engineering and Information \& Communication Technology (iCEEiCT), 2018, pp. 118-123.

[10] Rezoana Bente Arif, et al., "Study and Observation of the Variations of Accuracies for Handwritten Digits Recognition with Various Hidden Layers and Epochs using Convolutional Neural Network," in 2018 4th International Conference on Electrical Engineering and Information \& Communication Technology (iCEEiCT), 2018, pp. 112-117.

[11] Mohammad Mahmudur Rahman Khan, et al., "Study and Observation of the Variation of Accuracies of KNN, SVM, LMNN, ENN Algorithms on Eleven Different Datasets from UCI Machine Learning Repository," in 2018 4th International Conference on Electrical Engineering and Information \& Communication Technology (iCEEiCT), 2018, pp. 124-129.

[12] N. Wiener, et al., "Extrapolation, interpolation, and smoothing of stationary time series: with engineering applications," 1949.

[13] S. E. Reichenbach and J. Li, "Restoration and reconstruction from overlapping images for multi-image fusion," IEEE transactions on geoscience and remote sensing, vol. 39, pp. 769-780, 2001.

[14] M. S. Brown, et al., "Image pre-conditioning for out-of-focus projector blur," in Computer Vision and Pattern Recognition, 2006 IEEE Computer Society Conference on, 2006, pp. 19561963.

[15] Y.-M. Huang, et al., "Fast image restoration methods for impulse and Gaussian noises removal," IEEE Signal Processing Letters, vol. 16, pp. 457-460, 2009.

[16] H. Yamauchi and H.-P. Seidel, "Image restoration using multiresolution texture synthesis and image inpainting," in null, 2003, p. 120.
[17] S. W. Perry and L. Guan, "Weight assignment for adaptive image restoration by neural networks," IEEE Transactions on neural networks, vol. 11, pp. 156-170, 2000.

[18] C. V. Angelino, et al., "Image restoration using a knn-variant of the mean-shift," in Image Processing, 2008. ICIP 2008. 15th IEEE International Conference on, 2008, pp. 573-576.

[19] S. P. Awate and R. T. Whitaker, "Unsupervised, informationtheoretic, adaptive image filtering for image restoration," IEEE Transactions on Pattern Analysis \& Machine Intelligence, pp. 364-376, 2006.

[20] Y. Xiao, et al., "Restoration of images corrupted by mixed Gaussian-impulse noise via 11-10 minimization," Pattern Recognition, vol. 44, pp. 1708-1720, 2011.

[21] S. Lefkimmiatis, "Non-local color image denoising with convolutional neural networks," in Proc. IEEE Int. Conf. Computer Vision and Pattern Recognition, 2017, pp. 35873596.

[22] K. Zhang, et al., "Learning deep CNN denoiser prior for image restoration," in IEEE Conference on Computer Vision and Pattern Recognition, 2017.

[23] T. Tirer and R. Giryes, "Image restoration by iterative denoising and backward projections," IEEE Transactions on Image Processing, 2018.

[24] R. C. Gonzalez and R. E. Woods, "Digital image processing," ed: Prentice hall New Jersey, 2002.

[25] D. Kundur and D. Hatzinakos, "A novel blind deconvolution scheme for image restoration using recursive filtering," IEEE Transactions on Signal Processing, vol. 46, pp. 375-390, 1998.

[26] R. C. Gonzalez, et al., Digital Image Publishing Using MATLAB: Prentice Hall, 2004.

[27] M. Siddique, et al., "Implementation of Fuzzy C-Means and Possibilistic C-Means Clustering Algorithms, Cluster Tendency Analysis and Cluster Validation," arXiv preprint arXiv:1809.08417, 2018.

[28] Mohammad Mahmudur Rahman Khan, et al., "ADBSCAN: Adaptive Density-Based Spatial Clustering of Applications with Noise for Identifying Clusters with Varying Densities," in 2018 4th International Conference on Electrical Engineering and Information \& Communication Technology (iCEEiCT), 2018, pp. 107-111.

[29] R. G. Brown and P. Y. Hwang, "Introduction to random signals and applied Kalman filtering: with MATLAB exercises and solutions," Introduction to random signals and applied Kalman filtering: with MATLAB exercises and solutions, by Brown, Robert Grover.; Hwang, Patrick YC New York: Wiley, c1997., 1997.

[30] R. Grover and P. Y. Hwang, "Introduction to random signals and applied Kalman filtering," Willey, New York, 1992.

[31] A. Khireddine, et al., "Digital image restoration by Wiener filter in 2D case," Advances in Engineering Software, vol. 38, pp. 513-516, 2007.

[32] N. Kumar and K. K. Singh, "Wiener filter using digital image restoration," Int. J. Electron. Eng., vol. 3, pp. 345-348, 2011. 